\title{
Contribuição para o conhecimento da taxonomia, ecologia e fitogeografia de Briófitas da Amazônia Oriental
}

\author{
Lisi Dámaris Pereira ALVARENGA e Regina Célia Lobato LISBOA
}

\begin{abstract}
RESUMO
O presente trabalho documenta o inventário da brioflora da Estação Científica Ferreira Penna (ECFPn), localizada na FLONA Caxiuanã ( $1^{\circ} 42^{\prime} 30^{\prime \prime} S$ - 51 $\left.1^{\circ} 31^{\prime} 45^{\prime \prime W}\right)$, Melgaço - Pará, realizado por ocasião da primeira chamada do Programa de Residência em Estudos Amazônicos, iniciativa do Museu Paraense Emílio Goeldi. Musgos e Hepáticas foram amostrados nos ecossistemas de mata de terra firme, várzea, igapó, capoeiras e campina, e analisadas quanto à composição, riqueza e diversidade. Foram registradas 1081 ocorrências de briófitas de 120 espécies, sendo 79 hepáticas e 41 musgos, das quais oito hepáticas são novas referências para o Estado do Pará. As famílias de maior riqueza e assiduidade foram Lejeuneaceae (58 spp.), Calymperaceae (13 spp.), Sematophyllaceae (9 spp.) e Plagiochilaceae (7 spp.). As comunidades que melhor se fizeram representar foram a epífita (97 espécies/ 565 ocorrências) e a epíxila (65 spp./ 242 ocorr.), seguidas pelas comunidades epífila (27 spp./ 174 ocorr.) e terrícola (15 spp./ 96 ocorr.) e o ecossistema que apresentou maior riqueza e diversidade foi mata de terra firme. Quanto à composição nos substratos inventariados, foi observada uma inversão gradativa na proporção de riqueza entre hepáticas/ musgos, sendo as primeiras fortemente dominantes em folha, moderadamente dominantes em tronco vivo e morto, ao passo que musgos predominaram nos demais. Os resultados obtidos neste inventário, inclusive as novas referências para o Estado, contribuem para o entendimento dos padrōes de riqueza e diversidade na Floresta Amazônica e enriquecem a flora conhecida para o Estado do Pará.
\end{abstract}

PaLAVRAS-CHAVE: Caxiuanã, Estação Científica Ferreira Penna, musgos, hepáticas, inventário, Floresta Amazônica.

\section{Contribution to the taxonomy, ecology and phytogeographical knowledge of Bryophytes from Eastern Amazonia}

\begin{abstract}
This paper presents the floristic survey of Bryophytes from Ferreira Penna Scientific Station (ECFPn), located in the FLONA Caxiuanã (1'42’30”S - 51³1’45”W), Melgaço - Pará, that was undertaken in the first stage of the Residence Program in Amazonian Studies, a project of the Museu Paraense Emílio Goeldi. Mosses and liverworts were collected in non-flooded forest (terra firme), flooded forest (várzea and igapó), secondary forest and savanna ecosystems. They were analyzed for composition, richness and diversity. One thousand eighty one occurrences of 120 Bryophytes species (79 liverworts and 41 mosses) were recorded; and among them eight liverworts are new references from the state of Pará. The richest and most frequently encountered families were Lejeuneaceae (58 spp.), Calymperaceae (13 spp.), Sematophyllaceae (9 spp.) and Plagiochilaceae (7 spp.). The most representative communities were the epiphytic (97 species/ 565 occurrences) and epixylic (65 spp./ 242 ocurr.), followed by foliicolous (27 spp./ 174 ocurr.) and terricolous (15 spp./ 96 ocurr.); and the richest and most diverse ecosystem was the terra firme forest. The composition analysis of the surveyed substrates showed a gradual inversion in the richness ratio between liverworts/mosses, where the liverworts were strongly dominant in leaves, slightly dominant in live and dead trunks and the mosses were dominant in the others substrates. The results of this study, like the new references, contribute for understanding patterns of richness and diversity in Amazonia and increase the bryophyte flora of Pará.
\end{abstract}

KEY WORDS: Caxiuanã, Ferreira Penna Scientific Station, Mosses, Liverworts, Amazonian Forest

1 Universidade Federal de Pernambuco, CCB, Dpto. de Botânica, rua Prof. Moraes Rego S/N, Cidade Universitária, CEP 50670-420, Recife - PE. lisidamaris@yahoo.com.br

2 MCT-Museu Paraense Emílio Goeldi, Dpto. de Botânica, Caixa Postal 399. 660417-970. Belém - Pará. regina@museu-goeldi.br 


\section{INTRODUÇÃO}

O programa de Residência em Estudos Amazônicos foi uma iniciativa do Museu Paraense Emílio Goeldi (MPEG) que teve por objetivo incentivar a formação de profissionais em estudos de florestas tropicais. Para este fim, o programa forneceu estágios intensivos de três meses envolvendo atividades de campo e laboratório na estação Científica Ferreira Penna (ECFPn), localizada na Floresta Nacional de Caxiuanã, município de Melgaço - Pará (Amazônia Oriental) e no Núcleo de Pesquisa do MPEG, em Belém - Pará. O treinamento envolveu o aprimoramento de metodologias de pesquisa em florestas tropicais úmidas, incluindo coleta, tratamento do material coletado, identificação de espécimes para coleções científicas, tratamento de dados e elaboração de relatórios.

A primeira chamada do programa de Residência ocorreu no segundo semestre de 2004 e enfocou Briófitas, Pteridófitas e Fungos. A Dra. Regina Célia Tavares Lobato, pesquisadora e docente do Departamento de Botânica do MPEG foi a coordenadora do grupo de Briófitas, tratado neste trabalho. Foram estabelecidos como objetivos realizar o levantamento bibliográfico concernente à ecologia, taxonomia e fitogeografia de Briófitas nos biomas brasileiros, enfatizando primariamente a Floresta Amazônica, reconhecer e caracterizar os diferentes ecossistemas encontrados na área da Estação Científica Ferreira Penna, a saber, mata de terra firme, mata de inundação (várzea e igapó), vegetação secundária (capoeiras) e vegetação savanóide (campina), e comparar a composição, riqueza e diversidade das comunidades de briófitas presentes neles.

Os objetivos deste trabalho são apresentar e discutir os resultados alcançados durante as atividades do Programa de Residência e destacar sua contribuição para o conhecimento da brioflora amazônica.

\section{MATERIAL E MÉTODOS}

\section{ÁREA DE ESTUDO}

A área de estudo está localizada na ECFPn, instalada na Floresta Nacional de Caxiuanã (Flona Caxiuanã, 142'30"S - 5131'45”W), município de Melgaço, Pará. A ECFPn foi criada no final da década de 80 do século passado graças à colaboração de pesquisadores do Museu Goeldi e do botânico britânico Dr. Ghillean Tolmie Prance e sua equipe (Lisboa, P., 2002a). O convênio entre o Museu e o Instituto Brasileiro de Meio Ambiente e Recursos Renováveis (IBAMA) em 1990 estabeleceu que a área cedida à ECFPn seria de 33.000 hectares, correspondentes a $10 \%$ da extensão da FLONA Caxiuanã. Desde então inúmeros trabalhos científicos têm sido desenvolvidos na área possibilitando conclusões de monografias, dissertaçóes e teses de cientistas de diversas instituições. A significativa quantidade de informações resultantes de tais trabalhos foi sintetizada em Lisboa, P. (1997) e Lisboa, P. (2002b).

O relevo da área em que se localiza a FLONA Caxiuanã é plano e ondulado, sem grandes elevações, e os solos são da unidade de mapeamento LA11 Latossolos Amarelos Distróficos, variando apenas na textura (BRASIL, 1974 apud Ilkiu - Borges, 2000). A drenagem principal da região é composta pelo rio Anapu e pela baía de Caxiuanã, que possuem rios de águas pretas (Lisboa, P., 2002a). De acordo com a classificação de Köppen, o clima é do tipo AM ${ }^{\mathrm{i}}$, tropical úmido, com precipitação pluviométrica mais intensa de janeiro a março e, de acordo com dados coletados na base física da ECFPn, temperatura média anual de $26^{\circ} \mathrm{C}$ (Lisboa, P., 2002b).

A cobertura vegetal em Caxiuanã apresenta ecossistemas típicos da floresta amazônica, possuindo áreas de mata de terra firme, mata de inundação (várzea e igapó) trechos de vegetação secundária (capoeira) de diversas idades e trechos de vegetação savanóide (campinas amazônicas) (Lisboa, P., 2002b). As peculiaridades destas formações são brevemente resumidas a seguir de acordo com Ilkiu - Borges (2000) e Lisboa, P. (2002b):

Mata de terra firme - É o ecossistema mais extenso e diverso da área, correspondendo a ca. $85 \%$ da FLONA, e cresce sobre latossolos amarelos de origem terciária com textura argiloarenosa, ácidos, profundos, e oligotróficos.

Mata de inundação - 1) Mata de várzea - É localizada em áreas de inundação onde há flutuação do nível de água de até $30 \mathrm{~cm}$ diários, devido ao fluxo das marés e pela variação sazonal, ambos pouco conspícuos, e cresce sobre solos de aluvião quaternários, ricos em nutrientes; 2) Mata de igapó - É relativamente mais baixa e de menos complexidade e com menor biomassa em comparação com a floresta de terra firme e de várzea. Assim como a mata de várzea, a de igapó é exposta à alagação, porém de caráter mais permanente, em rios mais internos. Cresce em solos hidromórficos, ácidos e oligotróficos.

Vegetação secundária (capoeiras) - Estão presentes na ECFPn em manchas de até 5 hectares, resultantes da ação antrópica e com idades de até 50 anos e vegetação residual em sítios de pomares.

Vegetação savanóide (campinas amazônicas) - Apresenta características de cerrado, com estrato herbáceo contínuo, destacando-se as gramíneas, e arbustos e árvores baixas de aspecto tortuoso e escleromórfico, crescendo sobre solos hidromórficos e argilosos de origem quaternária.

\section{COLETA E IDENTIFICAÇÃO DE BRIÓFITAS}

As coletas foram feitas em todos os ecossistemas acima citados. Conforme acordado entre o projeto TEAM (Tropical Ecology, Assesmetn e Monitoring) e a coordenação do 
Programa de Residência, as coletas incluíram expedições aos plots permanentes do mencionado projeto. Estes são em número de seis (100 hectares cada) dispostos estrategicamente na ECFPn de forma aproximadamente eqüidistante e geralmente em mata de terra firme. Cada plot possui um quadrante central de 1 ha dentro do qual todas as árvores de DAP acima de $50 \mathrm{~cm}$ estão identificadas taxonomicamente e numeradas. As coletas foram procedidas ao longo das trilhas (geralmente apenas naquelas de melhor acesso ao quadrante central) e nos quadrantes centrais.

As coletas de briófitas foram realizadas de acordo com Lisboa (1993). Os substratos amostrados foram: folha (3-5 folhas, por forófito, mais velhas e/ ou com maior diversidade de briófitas, de arbustos e árvores jovens de até $2 \mathrm{~m}$ de altura), tronco vivo (uma amostra por forófito de ca. $10 \mathrm{~cm}^{2}$ de briófitas presentes até a altura de $2 \mathrm{~m}$ no tronco), base (= nível do solo) ou raiz de tronco vivo, tronco morto e solo (Gradstein et al., 1996; Kuusinen, 1996). Base, ou raiz de tronco vivo, foi considerado um substrato distinto apenas com o objetivo de comparar a composição das briofloras e verificar se há um padrão de sucessão ou mudança gradativa ao longo dos substratos em questão. Em adição a estes foram amostrados substratos especiais, isto é, de mais rara ocorrência, como cupinzeiro e concreto (rocha, solo pavimentado). Em seguida à coleta, o material era seco dentro dos sacos ao sol ou quando necessário, em caso de material muito úmido, como briófitas em galhos submersos, em estufa de campo $\left(40-60^{\circ} \mathrm{C}\right)$. Ao todo foram coletadas 423 amostras de briófitas.

Para fins de aplicabilidade nos testes estatísticos, o esforço amostral foi aproximadamente o mesmo para os locais de maior similaridade estrutural, isto é, os plots, bem como para os substratos presentes com relativamente elevada abundância nestes locais (Zar, 1996; krebs, 1989). Assim, foram coletadas em torno de 55 amostras em cada plot (incluindo trilhas e plots centrais) (Tabela 1). No tratamento dos dados, a nomeclatura adotada para as comunidades segue Gradstein et al. (2001) (comunidade - substrato): epífila - folha; epífita - tronco vivo; epíxila - tronco morto; terrícola - solo.

Para a identificação dos táxons foi utilizada, basicamente, a seguinte bibliografia: Florschütz (1964), Gradstein (1994), Buck (1998), Gradstein et al. (2001) e Gradstein e Costa (2003). A distribuição geográfica das espécies foi analisada apenas para hepáticas com base primariamente em Gradstein e Costa (2003). O sistema de classificação seguido está de acordo com Crandall - Stotler e Stotler (2000) para hepáticas e Buck e Goffinet (2000) para musgos. Todas as 423 amostras estão depositadas no herbário "João Murça Pires" (MG) do MPEG.

Tabela 1. Número de excursões e de amostras coletadas nos locais visitados durante a estadia na Estação Científica Ferreira Penna, Melgaço - PA. (P. central $=$ plot central).

\begin{tabular}{|c|c|c|c|}
\hline LOCAL & EXCURSÕES & AMOSTRAS COLETADAS & COORDENADAS GEOGRÁFICAS \\
\hline \multirow[t]{2}{*}{ Plot 1} & 4 & Trilha - 28 & - \\
\hline & & P. central - 30 & $01^{\circ} 42^{\prime} 49,1^{\prime \prime}$ S e $051^{\circ} 27^{\prime} 12^{\prime \prime} W$ \\
\hline \multirow[t]{2}{*}{ Plot 2} & 1 & Trilha - 27 & $01^{\circ} 44^{\prime} 30,6^{\prime \prime}$ S e $051^{\circ} 29^{\prime} 36,5^{\prime \prime} \mathrm{W}$ \\
\hline & & P. central - 26 & $01^{\circ} 43^{\prime} 43,3^{\prime \prime S}$ e $051^{\circ} 29^{\prime} 5 " W$ \\
\hline \multirow[t]{2}{*}{ Plot 3} & 1 & Trilha - 30 & - \\
\hline & & P. central - 29 & $01^{\circ} 44^{\prime} 5,6^{\prime \prime}$ S e $051^{\circ} 30^{\prime} 21,9^{\prime \prime} \mathrm{W}$ \\
\hline \multirow[t]{2}{*}{ Plot 4} & 3 & Trilha - 26 & $01^{\circ} 45^{\prime} 58,2^{\prime \prime}$ S e $051^{\circ} 31^{\prime} 22,3^{\prime \prime} \mathrm{W}$ \\
\hline & & P. central - 27 & $01^{\circ} 45^{\prime} 21,1^{\prime \prime}$ S e $051^{\circ} 31^{\prime} 19,1^{\prime \prime} \mathrm{W}$ \\
\hline \multirow[t]{2}{*}{ Plot 5} & 1 & Trilha - 28 & - \\
\hline & & P. central - 25 & $01^{\circ} 47^{\prime} 50,9^{\prime \prime S}$ e $051^{\circ} 35^{\prime} 26,5^{\prime \prime} W$ \\
\hline \multirow[t]{2}{*}{ Plot 6} & 1 & Trilha - 30 & $01^{\circ} 44^{\prime} 9,7^{\prime \prime}$ S e $051^{\circ} 25^{\prime} 53,4^{\prime \prime W}$ \\
\hline & & P. central - 32 & $01^{\circ} 43^{\prime} 22,7^{\prime \prime}$ S e $051^{\circ} 25^{\prime} 51,7^{\prime \prime} \mathrm{W}$ \\
\hline Igarapé Curuá & 1 & 15 & $01^{\circ} 42^{\prime} 53,3^{\prime \prime}$ S e $051^{\circ} 27^{\prime} 07,9^{\prime \prime} \mathrm{W}$ \\
\hline Igarapé Curuazinho & 1 & 10 & $01^{\circ} 44^{\prime} 07,2^{\prime \prime S}$ e $051^{\circ} 25^{\prime} 59,9^{\prime \prime} \mathrm{W}$ \\
\hline Igarapé Laranjal & 1 & 16 & $1^{0} 39^{\prime} 37,3^{\prime \prime}$ S e $51^{\circ} 20^{\prime} 4,11^{\prime \prime} W$ \\
\hline Igarapé Sta Rosa & 1 & 6 & $01^{\circ} 44^{\prime} 28^{\prime \prime}$ e e 051023'45”W \\
\hline Praia do Lisboa & 1 & 11 & $01^{\circ} 46^{\prime} 41^{\prime \prime}$ S e $051^{\circ} 25^{\prime} 25,6^{\prime \prime} \mathrm{W}$ \\
\hline Campina & 1 & 13 & $01^{\circ} 44^{\prime} 42,3^{\prime \prime}$ S e $051^{\circ} 23^{\prime} 10^{\prime \prime} \mathrm{W}$ \\
\hline Trilha torre de observação & 1 & 10 & $01^{\circ} 44^{\prime} 29,3^{\prime \prime}$ S e $051^{\circ} 27^{\prime} 33,5^{\prime \prime} \mathrm{W}$ \\
\hline Terra preta & 1 & 3 & - \\
\hline Heliponto & 1 & 1 & $01^{\circ} 44^{\prime} 18,6^{\prime \prime}$ S e $051^{\circ} 26^{\prime} 44^{\prime \prime W}$ \\
\hline Total & 20 & 423 & - \\
\hline
\end{tabular}




\section{ANÁLISE DOS DADOS}

A riqueza específica corresponde ao número de espécies encontrado para cada localidade e comunidade. A diversidade das briofloras nos plots e nos ecossistemas foi calculada através do índice de Shannon-Wiener (H'), conforme descrito em Ricklefs (1996), que analisa tanto o número de espécies como a equitabilidade entre elas (Krebs, 1989), sendo considerada como abundância relativa a freqüência das populaçōes de cada espécie de indivíduos, pois o tamanho e a natureza fragmentária das briófitas impossibilita a contagem de indivíduos (Bates, 1982). O cálculo da diversidade foi realizado através do software Programs for Ecological Methodology.

Para comparar as briofloras foram elaboradas matrizes de dados binários com dados qualitativos (presença/ ausência de espécies) para submisão ao índice de Sorensen (Zar, 1996). Os valores de similaridade foram submetidos à análise de agrupamento pelo método de agrupamento UPGMA (Unweighted Pair Group Method with Arithmetic Mean) com auxílio do Software NTSYSpc 2.1 (2000-2001, Applied Biostatistics Inc.). Para testar se os índices de similaridade $\mathrm{e}$, conseqüentemente, o agrupamento entre os fragmentos poderiam ser explicados pelo acaso, foram realizadas 2000 permutaçōes com o Software RandMat ver. 1.0 for Windows (http://eco.ib.usp.br/labmar - 05/2004).

\section{RESULTADOS E DISCUSSÃO}

\section{BRIOFLORA IDENTIFICADA}

Foram registradas 1081 ocorrências de briófitas nas 423 amostras coletadas na ECFPn, sendo identificadas ao todo 120 espécies consistindo de 79 hepáticas (Divisão MARCHANTIOPHYTA) e 41 musgos (Divisão BRYOPHYTA), das quais oito hepáticas são referidas pela primeira vez para o estado do Pará. Estas novas referências estão tratadas detalhadamente em Alvarenga et al. (2007). A seguir é apresentada a lista das espécies identificadas (as que estão assinaladas com asterisco são as novas referências para o estado do Pará).

\section{MARCHANTIOPHYTA}

\section{ANEURACEAE}

Riccardia sp.

\section{METZGERIACEAE}

Metzgeria cf. decipiens (C. Massal) Schiffn. \& Gottsche

\section{GEOCALYCACEAE}

Lophocolea liebmanniana Gottsche

Lophocolea perissodonta (Spruce) Steph.

\section{PLAGIOCHILACEAE}

* Plagiochila aerea Tayl.
Plagiochila disticha (Lehm. \& Lindenb.) Lindenb.

Plagiochila distinctifolia Lindenb.

* Plagiochila gymnocalycina (Lehm. \& Lindenb.) Lindenb.

Plagiochila cf. lingua Steph.

* Plagiochila martiana (Nees) Lindenb.

Plagiochila montagnei Nees

\section{CALYPOGEIACEAE}

Calypogeia laxa Gottsche \& Lindenb.

\section{LEPIDOZIACEAE}

*Arachniopsis monodactyla (Spruce) R.M. Schust.

Micropterygium sp.

Micropterygium cf. pterygophyllum (Nees) Trevis

Micropterygium cf. trachyphyllum Reimers

\section{JUBULACEAE}

Frullania gibbosa Nees

Frullania nodulosa (Reinw. et al.) Nees

\section{LEJEUNEACEAE}

Acrolejeunea torulosa (Lehm. \& Lindenb.) Schiffn.

Aphanolejeunea gracilis Jovet - Ast

Aphanolejeunea truncatifolia Horik.

Aphanolejeunea winkleri M.I.Morales \& A.Lücking

Archilejeunea auberiana (Mont.) A.Evans

Archilejeunea fuscescens (Hampe ex Lehm.) Fulford

Archilejeunea parviflora (Nees) Schiffn.

Ceratolejeunea coarina (Gottsche) Steph.

Ceratolejeunea cornuta (Lindenb.) Schiffn.

Ceratolejeunea cubensis (Mont.) Schiffn.

* Ceratolejeunea desciscens (Sande Lac.) Steph.

Ceratolejeunea guianensis (Nees \& Mont.) Steph.

Ceratolejeunea laetefusca (Austin) R.M. Schust.

Ceratolejeunea minuta Dauphin

Cheilolejeunea adnata (Kunze ex Lehm.) Grolle

Cheilolejeunea holostipa (Spruce) Grolle \& R.-L. Zhu

Cheilolejeunea rigidula (Nees \& Mont.) R.M.Schust.

Cheilolejeunea trifaria (Reinw., Blume e Nees) Mizut.

Cololejeunea obliqua (Nees \& Mont.) Schiffn.

Cololejeunea surinamensis Tixier

${ }^{*}$ Colura greig-smithii Jovet - Ast

Colura tortifolia (Nees \& Mont.) Steph.

Cyclolejeunea convexistipa (Lehm. \& Lindenb.) A.Evans

Cyclolejeunea luteola (Spruce) Grolle 
Diplasiolejeunea brunnea Steph.

Drepanolejeunea polyrhiza (Nees) Grolle \& R.-L. Zhu

Haplolejeunea cucullata (Steph.) Grolle

Harpalejeunea oxyphylla (Nees \& Mont.) Steph.

Harpalejeunea stricta (Lindenb. \& Gottsche) Steph.

Lejeunea boryana Mont.

Lejeunea caespitosa Lindenb.

Lejeunea controversa Gottsche

Lejeunea flava (Sw.) Nees

Lejeunea glaucescens Gottsche

Lejeunea laetevirens Nees \& Mont.

* Lejeunea raddiana Lindenb.

Lejeunea tapajosensis Spruce

Lejeunea sp.

Leptolejeunea elliptica (Lehm. \& Lindenb.) Schiffn.

Leptolejeunea tridentata Bischl.

Lopholejeunea subfusca (Nees) Schiffn.

Microlejeunea epiphylla Bischl.

Neurolejeunea seminervis (Spruce) Schiffn.

Pictolejeunea picta (Gottsche ex Steph.) Grolle

Prionolejeunea denticulata (F.Weber) Schiffn.

Prionolejeunea muricato-serrulata (Spruce) Steph.

Pycnolejeunea contigua (Nees) Grolle

Pycnolejeunea macroloba (Nees \& Mont.) Schiffn.

Rectolejeunea berteroana (Gottsche ex Steph.) A.Evans

* Rectolejeunea emarginuliflora (Gottsche) A.Evans

Stictolejeunea squamata (Willd. ex Web.) Schiffn.

Symbiezidium barbiflorum (Lindenb. \& Gottsche) A.Evans

Symbiezidium transversale (Sw.) Trevis

Thysananthus amazonicus (Spruce) Schiffn.

Trachylejeunea anaeogyna (Spruce) Grolle

Trachylejeunea sp.

Xylolejeunea crenata (Nees \& Mont.) X.-L. He

Xylolejeunea sp.

9. RADULACEAE

Radula flaccida Lindenb.

Radula javanica Gottsche

Radula recubans Tayl.

\section{BRYOPHYTA}

\section{FISSIDENTACEAE}

Fissidens guianensis Mont.
Fissidens prionodes Brid.

\section{LEUCOBRYACEAE}

Leucobryum martianum (Hornsch.) Hampe ex Müll. Hal.

Ocrobryum sp.

\section{POTTIACEAE}

Hyophila sp.

Pottiaceae sp.

\section{CALYMPERACEAE}

Calymperes afzelii Schwägr.

Calymperes lonchophyllum Schwägr.

Calymperes palisotii Schwägr.

Calymperes platyloma Mitt.

Calymperes uleanum Broth.

Octoblepharum albidum Hedw.

Octoblepharum cylindricum Schimp. ex Mont.

Octoblepharum pulvinatum (Dozy \& Molk.) Mitt.

Syrrhopodon cryptocarpos Dozy \& Molk.

Syrrhopodon incompletus Schwägr.

Syrrhopodon ligulatus Mont.

Syrrhopodon parasiticus (Brid.) Besch.

Syrrhopodon simmondsii Steere

14. ORTOTRICHACEAE

Groutiella obtusa (Mitt.) Müll. Hal.

\section{PHYLLODREPANIACEAE}

Mniomalia virides (Mitt.) Müll. Hal.

\section{LEUCOMIACEAE}

Leucomium strumosum (Hornsch.) Mitt.

\section{PILOTRICHACEAE}

Callicostella rufescens (Mitt.) A.Jaeger

Callicostella pallida (Hornsch.) Ångstr.

Lepidopilum surinamense Müll. Hal.

Pilotrichum evanescens Müll. Hal.

\section{BRACHYTHECIACEAE}

Zelometeorium patulum (Hedw.) Manuel

19. HYPNACEAE

Isopterygium tenerum (Schwägr.) Mitt.

\section{NECKERACEAE}

Neckeropsis undulata (Hedw.) Reich.

\section{SEMATOPHYLLACEAE}

Meiothecium boryanum (Müll. Hal.) Mitt. 
Potamium lonchophyllum (Mont.) Mitt.

Sematophyllum subpinnatum (Brid.) Britt.

Sematophyllum subsimplex (Hedw.) Mitt.

Taxithelium planum (Brid.) Mitt.

Taxithelium pluripunctatum (Renauld \& Cardot) W.R. Buck

Trichosteleum fluviale (Mitt.) Broth.

Trichosteleum intrincatum (Thér.) A.Jaeger

Trichosteleum papillosum (Hornsch.) A. Jaeger

\section{STEREOPHYLLACEAE}

Pilosium chlorophyllum (Hornsch.) Müll. Hal.

\section{THUIDIACEAE}

Pelekium scabrosulum (Hedw.) Touw

Pelekium schistocalyx (Müll. Hal.) Touw

\section{RIQUEZA E COMPOSIÇÃO}

A riqueza específica registrada para a área estudada na ECFPn foi de 120 espécies ao todo. Hepáticas predominaram sobre musgos em riqueza específica, sendo esta distribuída em um menor número de famílias (Tabela 2). Dentre as 23 famílias assinaladas, as de maior riqueza específica foram Lejeuneaceae (58 esp.), Calymperaceae (13 esp.), Sematophyllaceae (9 esp.) e Plagiochilaceae (7 esp.). Em termos de frequêencia, tal padrão de predominância entre hepáticas (624 ocorrências) e musgos (457) bem como dentre as famílias se repete, com destaque ainda para Fissidentaceae dentre os musgos (Figura 1).

Tabela 2 - Riqueza de famílias, gêneros e espécies das divisões Marchantiophyta e Bryophyta registradas para a Estação Científica Ferreira Penna, Melgaço - PA.

\begin{tabular}{lccc}
\hline & FAMÍLIAS & GÊNEROS & ESPÉCIES \\
\hline Bryophyta & 14 & 24 & 41 \\
Marchantiophyta & 9 & 35 & 79 \\
Total & 23 & 59 & 120 \\
\hline
\end{tabular}

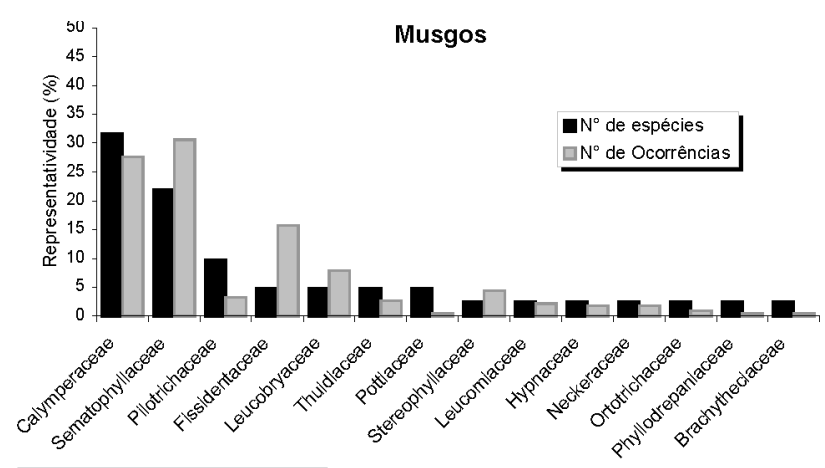

Figura 1a - Representatividade das famílias registradas para a ECFPn, Melgaço - PA.

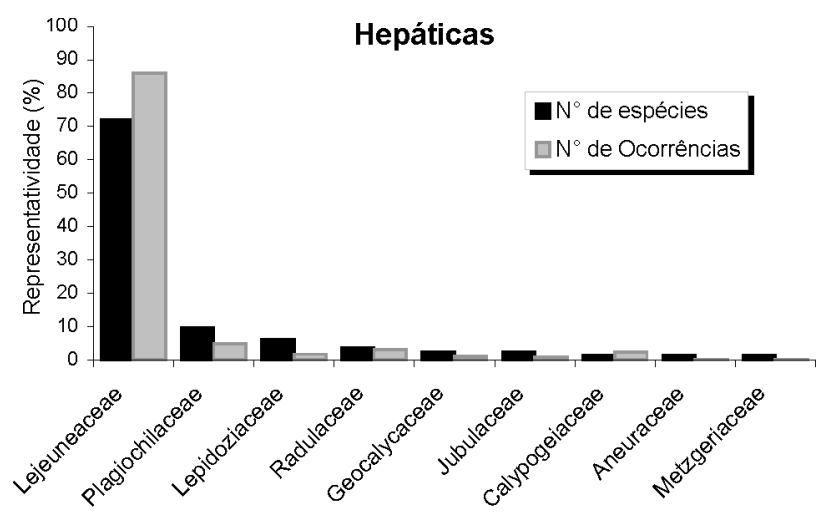

Figura 1b - Representatividade das famílias registradas para a ECFPn, Melgaço - PA.

Estes valores estão de acordo com o esperado, pois conforme menciona Richards (1984), em florestas tropicais de planície, de baixas altitudes, como é o caso da Amazônia, a riqueza específica de hepáticas é sempre maior que a de musgos. Este autor cita as observaçōes de Spruce em 1853 sobre as florestas na Amazônia, numa grande área em Rio Negro. Spruce disse "eu não tenho encontrado mais musgos nesta área do que eu encontraria numa similar na Europa". A riqueza de musgos em áreas tropicais parece ser mais saliente em florestas de maior altitude. Também esperado era o padrão de predominância das famílias encontrado. Gradstein e Pócs (1989) incluem estas famílias nas 15 detentoras de $90 \%$ das espécies ocorrentes em florestas tropicais.

A maioria das espécies ocorreu com frequiência rara, com uma a cinco ocorrências. Isto comprova que a norma para as fanerógamas de raridade natural (distribuição geográfica restrita) se repete para este grupo de criptógamos, norma, aliás, também para demais grupos biológicos em ecossistemas tropicais (Ricklefs, 1996; Kageyama e Lepsch-Cunha, 2001; Tabarelli et al., 2002).

\section{SUBSTRATOS}

As comunidades que melhor se fizeram representar foram a epífita (97 espécies/ 565 ocorrências) e a epíxila (65 esp./ 242 ocorr.), seguidas pelas comunidades epífila (27 esp./ 174 ocorr.) e terrícola (15 esp./ 96 ocorr.). Os substratos cupinzeiro e concreto apresentaram representatividade mais discreta: 1 esp./ 2 ocorr. e 3 esp./ 3 ocorr., respectivamente. Mesmo tomando em conta apenas as amostras coletadas acima da base do tronco vivo, posto que ao incluir as da base o total de amostras torna-se o dobro em relação aos demais substratos inventariados, a representatividade da comunidade epífita ainda é acentuada, com 85 espécies/ 336 ocorrências. Os substratos tronco vivo e tronco morto são apontados em literatura como sendo de fato os mais favoráveis para a colonização de briófitas de forma geral devido tanto à maior capacidade de retenção de umidade como à própria 
disponibilidade no ambiente (Richards, 1984; Germano e Pôrto, 1996). O substrato solo é disponível em menor quantidade, por exemplo, principalmente devido ao acúmulo de necromassa abundante em florestas tropicais (Ilkiu-Borges, 2000). Este substrato, juntamente com rocha e folha, impõe de certa forma determinadas barreiras ao estabelecimento e sobrevivência às populações de briófitas, levando a uma composição taxonomicamente mais restrita e especializada, isto é, com adaptaçōes específicas, em relação àqueles primeiros substratos (Richards, 1984; Gradstein, 1997).

Foi observada uma inversão gradativa na proporção de riqueza entre hepáticas/musgos nos substratos inventariados, sendo as primeiras fortemente dominantes em folha, moderadamente dominantes em tronco vivo e tronco morto, ao passo que musgos predominam nos demais (Figura 2). Em termos de freqüência, esta inversão se inicia na base de tronco vivo (Figura 2). Este padrão condiz com o mencionado em literatura para florestas tropicais (Richards, 1984; Germano e Pôrto, 1996; Gradstein, 1997).

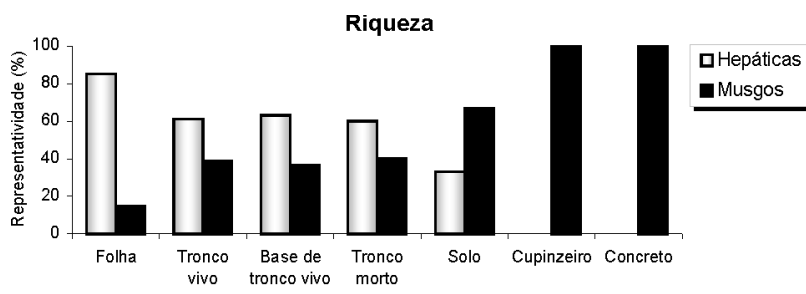

Figura 2a - Representatividade de musgos e hepáticas em termos de riqueza e ocorrências nos substratos inventariados na ECFPn, Melgaço - PA.

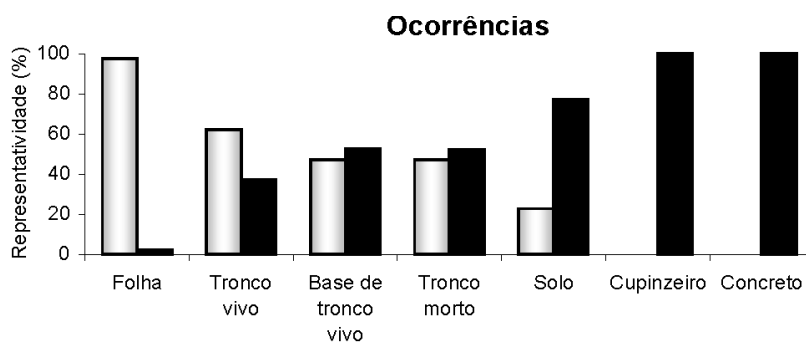

Figura $\mathbf{2 b}$ - Representatividade de musgos e hepáticas em termos de riqueza e ocorrências nos substratos inventariados na ECFPn, Melgaço - PA.

O estudo da similaridade entre os substratos confirmou a mudança gradativa também na composição de espécies, evidenciando ainda que a brioflora epífila e terrícola foram suficientemente distintas para gerar índices de similaridade com os demais grupos explicados apenas pelo acaso (Figura 3). A composição da brioflora nos substratos cupinzeiro e concreto apresentou os menores índices de similaridade não explicados pelo acaso. Isto ratifica a hipótese de que as peculiaridades destes substratos, folha, rocha e solo, levam a uma composição de brioflora mais restrita e especializada.

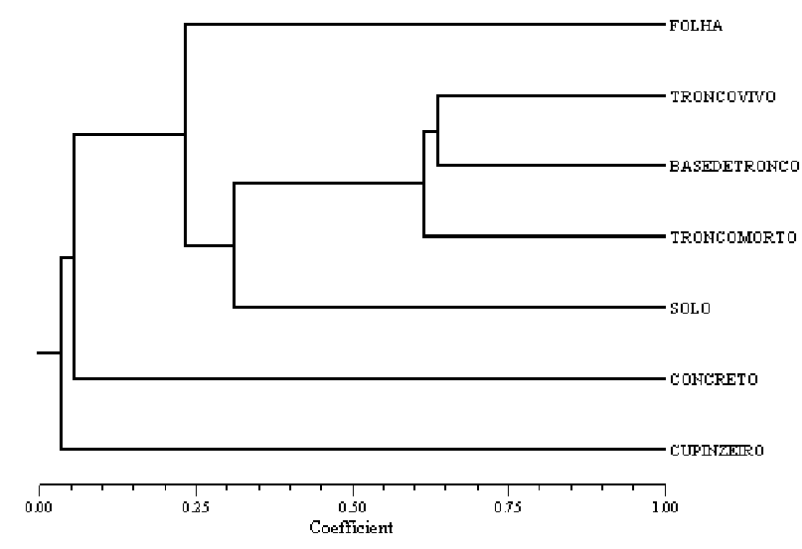

Figura 3 - Dendograma de similaridade das briofloras nos substratos inventariados na ECFPn Melgaço - PA. Limite de significância a partir do qual os valores dos índices podem ser considerados significativos: 0,27.

As famílias predominantes na comunidade epífila, avaliando riqueza específica e número de ocorrências, foram Lejeuneaceae e Radulaceae; na epífita, Lejeuneaceae, Calymperacae, Sematophyllaceae e Plagiochilaceae; na epíxila, Lejeuneaceae, Sematophyllaceae, Calymperaceae e Leucobryaceae; e na terrícola, Fissidentaceae e Calypogeiaceae. A predominância destas famílias em tais substratos é recorrente em literatura (Pócs, 1996; Gradstein, 1997; Lücking, 1997).

\section{ESTUDO COMPARATIVO DOS PLOTS DO PROJETO TEAM}

Os plots apresentaram riqueza e diversidade de espécies consideravelmente elevados, sendo os menores e os maiores valores encontrados para o plot 3 e o plot 5 , respectivamente (Figura 4). Estes dois plots também evidenciaram elevada similaridade de composição, embora esta possa ser explicada pelo acaso (Figura 5a).

É evidente, contudo, uma grande similaridade entre os plots ao analisar a composição dos substratos inventariados de todos os plots concomitantemente (Figura 5b). As comunidades

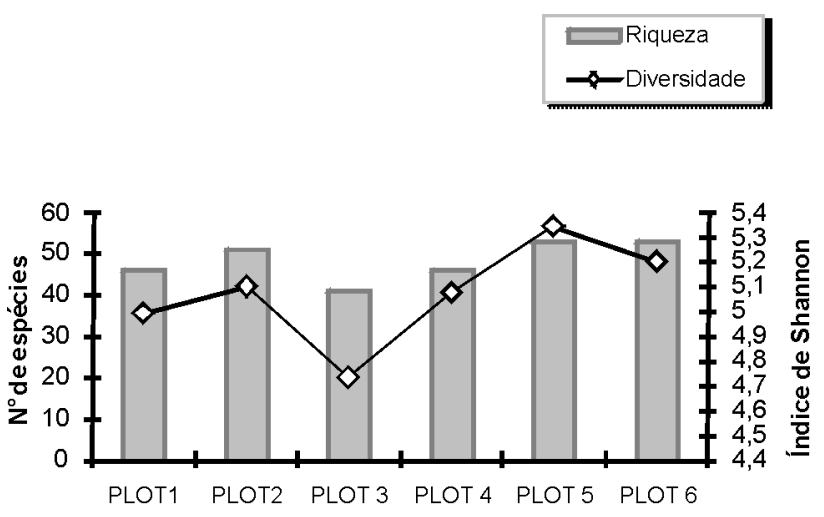

Figura 4 - Riqueza e diversidade dos plots do projeto TEAM inventariados na ECFPn, Melgaço - PA. 


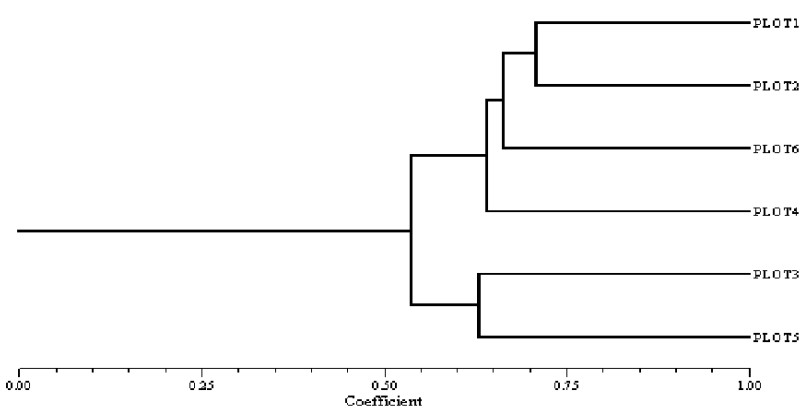

Figura $5 \mathbf{a}$ - Dendograma de similaridade das briofloras nos plots do projeto TEAM inventariados na ECFPn, Melgaço - PA. a) composição geral dos plots; b) composição dos substratos inventariados nos plots. Limite de significância a partir do qual os valores dos índices podem ser considerados significativos: a) 0,75 e b) 0,50 .

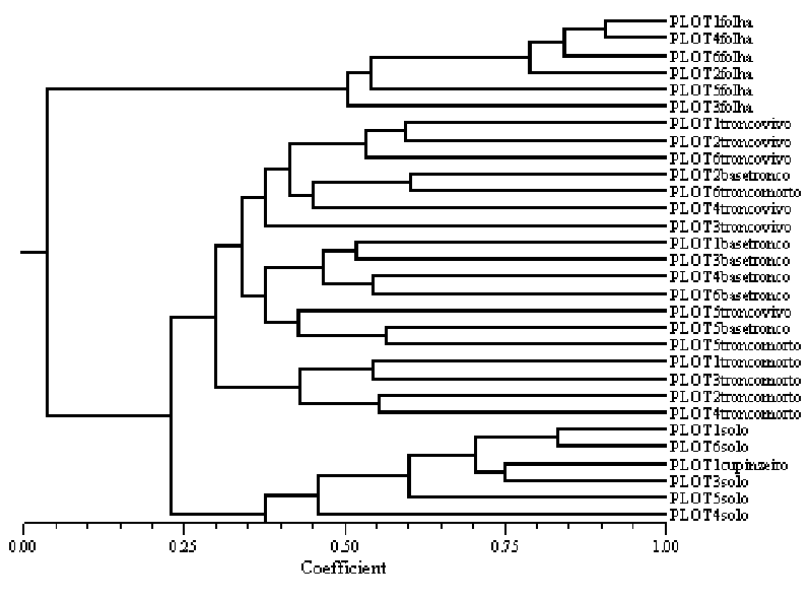

Figura $\mathbf{5 b}$ - Dendograma de similaridade das briofloras nos plots do projeto TEAM inventariados na ECFPn, Melgaço - PA. a) composição geral dos plots; b) composição dos substratos inventariados nos plots. Limite de significância a partir do qual os valores dos índices podem ser considerados significativos: a) 0,75 e b) 0,50 .

de todos os plots parecem ter elevada homogeneidade de composição, com índices elevados e significativos, o que é natural, pois os plots estão geralmente no mesmo ecossistema (mata de terra firme) e, assim, condiçôes microclimáticas muito similares.

\section{ESTUDO COMPARATIVO DOS ECOSSISTEMAS ESTUDADOS}

$\mathrm{O}$ ecossistema que apresentou maior riqueza e diversidade foi mata de terra firme seguido por mata de várzea (Figura 6), e a similaridade entre os ecossistemas decresceu no mesmo sentido que estes dois parâmetros (Figura 7). Estes resultados podem ser explicados em parte pelo número de amostras coletado em cada ecossistema, que decresceu no sentido acima mencionado, que reflete a própria disponibilidade de habitat.

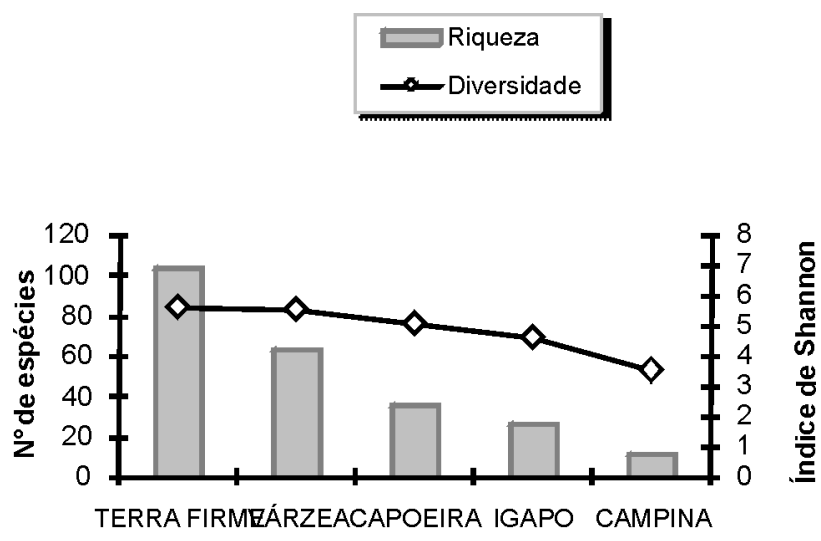

Figura 6 - Riqueza e diversidade da briofloras nos ecossistemas inventariados na ECFPn, Melgaço - PA.

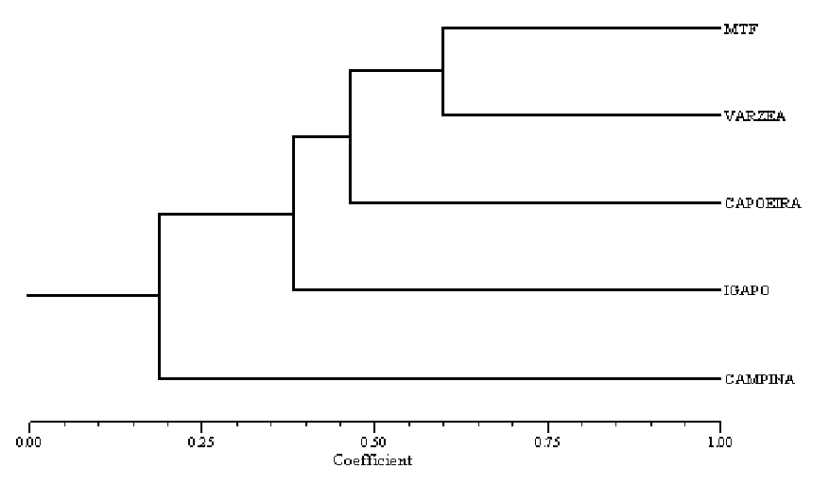

Figura 7 - Dendograma de similaridade das briofloras nos ecossistemas estudados na ECFPn, Melgaço - PA. Limite de significância: 0,50.

No estudo recentemente realizado na ECFPn por IlkiuBorges (2000) foi encontrada maior riqueza de espécies da família Lejeuneaceae (hepática) no ecossistema mata de várzea. Visto este ecossistema possuir umidade mais constante nos substratos, este resultado encontrado procede. Áreas onde a umidade ambiental é maior propiciam o estabelecimento de populaçôes de briófitas mais vastas e ricas (Gradstein et al., 2001). O resultado na presente pesquisa difere do de IlkiuBorges (2000) possivelmente em razão da inclusão dos musgos que possuem uma faixa de tolerância a ambientes secos mais ampla (Schofield, 1985).

\section{CONCLUSÕES}

Tendo em mente que o presente estudo consistiu de um inventário rápido (3 meses e ca. 400 amostras), fica evidente que a brioflora da ECFPn possui elevada riqueza e diversidade comparável àquela de estudos mais minuciosos e extensivos em outros biomas reconhecidamente ricos (Germano e Pôrto, 1996; 1998). Áreas sob algum tipo de pressão antrópica 
usualmente não sustentam uma brioflora rica mesmo na Floresta Amazônica (Lisboa e Ilkiu-Borges, 1995).

$\mathrm{O}$ assinalamento de oito táxons inéditos para o Estado (Alvarenga et al., 2007) é evidência de que o estudo cumpriu seu objetivo, que era primordialmente contribuir para o conhecimento da brioflora amazônica. O estudo, ademais, confirma que a brioflora da Amazônia abriga uma diversidade e riqueza dignas de serem melhor exploradas.

\section{AGRADECIMENTOS}

As autoras agradecem a Maria Aparecida, pesquisadora do Projeto TEAM pela orientação no trabalho de campo e à Coordenação da Estação Científica Ferreira Penna e do Programa de Residência em Estudos Amazônicos nas pessoas de Alcir Favacho e Luiza Magali Henrique, respectivamente.

\section{BIBLIOGRAFIA CITADA}

Alvarenga, L.D.P.; Lisboa, R.C.L.; Tavares, A.C.C. 2007. Novas Referências de Hepáticas (Marchantiophyta) da Floresta Nacional de Caxiuanã, para o Estado do Pará, Brasil. Acta Botanica Brasilica, 21(3): 240-248.

Bates, J.W. 1982. Quantitative Approaches in Bryophyte Ecology. In: Smith, A.J.E. (Ed.). Bryophyte Ecology. Chapman and Hall Ltd. London. p. 1-44.

Benzing, D.H. 1990. Vascular Epiphytes. Cambridge University Press, New York. 353pp.

Buck, W.R. 1998. Pleurocarpous Mosses of the West Indies. Memoirs of the New York Botanical Garden, 1: 1-401.

Buck, W.R.; Goffinet, B. 2000. Morphology and Classification of Mosses. In: Shaw, J.; Goffinet, B. (Eds.). Bryophyte Biology. Cambridge University Press, Cambridge. p. 71-123.

Florschütz, P.A. 1964. The mosses of Suriname. E.J. Brill, Leiden. $271 \mathrm{p}$.

Chang, S-C.; Lai, I-L.; Wu J-T. 2002. Estimation of Fog Deposition on Epiphytic Bryophytes in a Subtropical Montane Forest Ecosystem in Northeastern Taiwan. Atmosferic Research, 64: 159-167.

Coxon, D.S.; Nadkarni, N.M. 1995. Ecological roles of epiphytes in nutrient cycles of forest ecosystems. In: Lowman, M.D.; Nadkarni, N.M. (Eds). Forest Canopies. Academic Press, San Diego. p. 495-543.

Crandall-Stotler, B.; Stotler, R.E. 2000. Morphology and classification of Marchantiophyta. In: Shaw, J.; Goffinet, B. (Eds.). Bryophyte Biology. Cambridge University Press, Cambridge. p. 21-70.

Germano, S.R; Pôrto, K.C. 1996. Floristic survey of epixylic bryophytes of an area remnant of the Atlantic Forest (Timbaúba - PE, Brazil) 1. Hepaticopsida (except Lejeuneaceae) and Bryopsida. Tropical Bryology, 12: 21-28.

Germano, S.R.; Pôrto, K.C. 1998. Briófitas epíxilas de uma área remanescente de floresta atlântica (Timbaúba, PE, Brasil). 2. Lejeuneaceae. Acta Botanica Brasilica, 12(1): 53-66.
Gradstein, S.R. 1994. Lejeuneaceae; Ptychantheae, Brachiolejeuneae. Flora Neotropica Monograph, 62: 1-225.

Gradstein, S.R. 1997. The taxonomic diversity of epiphyllous Bryophytes. Abstracta Botanica, 21(1): 15-19.

Gradstein, S.R.; Churchill, S.P.; Salazar Allen., N. 2001. Guide to the Bryophytes of Tropical America. Memoirs of the New York Botanical Garden, 86: 1-577.

Gradstein, S.R.; Hietz, P.; Lücking, R.; Lücking, A.; Sipman, H.J.M; Vester, H.F.M; Wolf, J.H.D.; Gardette, E. 1996. How to sample the epiphytic diversity of tropical rain forests. Ecotropica, 2: 59-72.

Gradstein, S.R.; Costa, D.P. 2003. The Hepaticae and Anthocerotae of Brazil. Memoirs of the New York Botanical Garden, 88: $1-673$.

Gradstein, S.R.; Pócs, T. 1989. Bryophytes. In: Lieth, H.; Werger, M.J.A. (Eds.). Tropical Rain Forest Ecosystems. Elseiver Science Publishers, Amsterdam. p. 311-325.

Ilkiu-Borges, A.L. 2000. Lejeuneaceae (Hepaticae) da Estação Cientifica Ferreira Penna, Caxiuanã, município de Melgaço, Pará. Dissertação de mestrado em Agronomia. Faculdade de Ciências Agrárias do Pará, Belém.

Ilkiu-Borges, A.L.; Lisboa, R.C.L. 2004. Cololejeuneae (Lejeuneaceae, Hepaticae) na estação Científica Ferreira Pena, Melgaço, PA, Brasil. Acta Botanica Brasilica, 18(4): 887-902.

Kageyama, P.; Lepsch-Cunha, N.M. 2001. Singularidade da biodiversidade nos trópicos. In: Garay, I. E. G.; Dias, B. F. S. (Orgs.). Conservação da Biodiversidade em Ecossistemas Tropicais. Editora vozes, Rio de Janeiro. p. 199-214.

Krebs, C.J. 1989. Ecological methodology. Harper e Row Publishers, New York. 654p.

Kuusinen, M. 1996. Epiphyte flora and diversity on basal trunks of six old-growth forest tree species in southern and middle boreal Finland. Lichenologist, 28 (5): 443-463.

Lisboa, P.L.B. (org.) 1997. Caxiuanã. Belém, Museu Paraense Emílio Goeldi. 446p.

Lisboa, P.L.B. (org.) 2002a. Natureza, homem e manejo de recursos naturais na regiāo de Caxiuanã, Melgaço, Pará. Museu Paraense Emílio Goeldi, Belém. 237p.

Lisboa, P.L.B. (org.) 2002b. Caxiuanã: populaçôes tradicionais, meio físico e diversidade biológica. Belém, Museu Paraense Emílio Goeldi. 734p.

Lisboa, R.C.L. 1993. Musgos acrocárpicos do Estado de Rondônia. Museu Paraense Emílio Goeldi, Belém. 272p.

Lisboa, R.C.L. 1994. Adiçôes à brioflora do Estado do Pará. Boletim do Museu Paraense Emílio Goeldi, 10(1): 15-42.

Lisboa, R.C.L.; Ilkiu-Borges, A.L. 1995. Diversidade de briófitas de Belém (PA) e seu potencial como indicadoras de poluição urbana. Boletim do Museu Paraense Emílio Goeldi, 12: 161-181.

Lisboa, R.C.L.; Ilkiu-Borges, A.L. 1997. Novas ocorrências de Bryophyta (musgos) para o Estado do Pará, Brasil. Acta Amazonica, 27 (2): 81-102.

Lisboa, R.C.L.; Ilkiu-Borges, A.L. 2001. Briófitas de São Luís do Tapajós, município de Itaituba, com novas adições para o 
Estado do Pará. Boletim do Museu Paraense Emílio Goeldi, 17 (1): 75-91.

Lisboa, R.C.L.; Lima, M.J.L. 1997. Leucophanaceae, nova família de Bryophyta para o Pará, Brasil. Acta Botanica Brasilica, 11(1): 79-85.

Lücking A., 1997. Diversity and distribution of epiphyllous bryophytes in a tropical rainforest in Costa Rica. Abstracta Botanica, 21(10): 79-87.

Osakada, A.; Lisboa, R.C.L. 2004. Novas ocorrências de hepáticas (Marchantiophyta) para o Estado do Pará Brasil. Acta Amazônica, 34(2): 197-200.

Pócs, T. 1996. Epiphyllous Liverwort Diversity at Worldwide Level and its Threat and Conservation. Anales Instituto Biologia Universidad Nacional Autónoma México Ser. Bot., 67 (1): 109127.

Richards, P.W. 1984. The ecology of tropical forest bryophytes. In: Schuster, R.M. (Ed.). New Manual of Bryology. The Hattori Botanical Laboratory, Nichinan. p. 1233-1270.
Ricklefs, R.M. 1996. A Economia da Natureza. $3^{a}$ edição. Editora Guanabara Koogan S.A., Rio de Janeiro. 470p.

Schofield, W.B. 1985. Introduction to Bryology. MacMillan Publishing, New York. 418p.

Tabarelli, M., Marins, J. F.; Silva, J. M. C. 2002. La biodiversidad brasileña amenazada. Investigación y Ciencia, 308: 42-49.

Uniyal, P.L. 1999. Role of Bryophytes in Conservation of Ecosystems and Biodiversity. The Botanica, 49: 101-115.

Veneklaas, E.J.; Zagt, R.J.; Leerdam, A.; Ek, R.; Broekhoven, A.J.; Genderen, M. 1990. Hydrological properties of the epiphyte mass of a montane tropical rain forest, Colombia. Vegetatio, 89: 183-192.

Zar, J. H. 1996. Biostatistical Analysis. $4^{\text {th }}$ Edit. Prentice Hall, New Jersey. 803p.

Recebido em 23/11/2007

Aceito em 20/04/2009 